\title{
OPEN A systematic review and meta-analysis of effects of menopausal hormone therapy on cardiovascular diseases
}

\author{
Ji-Eun Kim ${ }^{1,2}$, Jae-Hyuck Chang ${ }^{3}$, Min-Ji Jeong ${ }^{3}$, Jaesung Choi ${ }^{1,2}$, JooYong Park ${ }^{1,2}$, \\ Chaewon Baek ${ }^{3}$, Aesun Shin ${ }^{4,5,6}$, Sang Min Park ${ }^{1,7}$, Daehee Kang ${ }^{4,5,6,8}$ \& Ji-Yeob Choi ${ }^{1,2,5,9 凶}$
}

A systematic review and meta-analysis of randomized controlled trials (RCTs) and observational studies was conducted to assess the association between menopausal hormone therapy and cardiovascular disease. The PubMed and EMBASE databases were searched for articles published from 2000 to 2019, using review methods based on a previous Cochrane review. Quality assessment of RCTs and observational studies was conducted using the Jadad scale and the Newcastle-Ottawa Scale, respectively. A total of 26 RCTs and 47 observational studies were identified. The study populations in the RCTs were older and had more underlying diseases than those in the observational studies. Increased risks of venous thromboembolism [summary estimate (SE), 95\% confidence interval (CI): RCTs, 1.70, 1.33-2.16; observational studies, 1.32, 1.13-1.54] were consistently identified in both study types, whereas an increased risk of stroke in RCTs (SE: 1.14, 95\% Cl: 1.04-1.25) and a decreased risk of myocardial infarction in observational studies (SE: $0.79,95 \% \mathrm{Cl}: 0.75-0.84$ ) were observed. Differential clinical effects depending on timing of initiation, underlying disease, regimen type, and route of administration were identified through subgroup analyses. These findings suggest that underlying disease and timing of initiation should be carefully considered before starting therapy in postmenopausal women.

Previous studies have reported that while premenopausal women have a lower risk of developing cardiovascular disease (CVD) than men, postmenopausal women have a higher risk ${ }^{1,2}$. It has been suggested that metabolic changes due to estrogen depletion after menopause lead to an increased CVD risk in postmenopausal women ${ }^{3-5}$. Experimental studies have identified several protective mechanisms of estrogen against CVD, which include increasing angiogenesis and vasodilation, and reducing fibrosis and oxidative stress ${ }^{6}$. Menopausal hormone therapy (MHT) was suggested to contribute to the reduction of CVD risk based on the hypothesis of cardioprotection by estrogen ${ }^{7,8}$. Many randomized controlled trials (RCTs) and observational studies have investigated the association between MHT and CVD risk; however, there have been inconsistent results among studies. Early observational studies have reported beneficial effects of MHT on CVD, whereas large RCTs, such as the Women's Health Initiative (WHI) and the Heart and Estrogen/Progestin Replacement Study (HERS) have not ${ }^{7-11}$. However, some major limitations of the RCTs are that the women were older, had initiated MHT late after menopause, and either had CVD risk factors at baseline or had a history of a CVD event ${ }^{10-12}$. Since the publication of the WHI report, several studies have reevaluated the risk profile of $\mathrm{MHT}^{13-15}$. Despite that, controversies remain regarding CVD-related risks and benefits of MHT. Therefore, emphasis has been placed on the necessity of additional studies that evaluate the following factors: dose of estrogen, route of administration, timing after menopause, duration of use, other hormone effects, pre-existing pathology, and age ${ }^{3}$.

${ }^{1}$ Department of Biomedical Sciences, Seoul National University Graduate School, 103 Daehak-ro, Jongno-gu, Seoul 03080, Korea. 'BK21plus Biomedical Science Project, Seoul National University College of Medicine, Seoul, Korea. ${ }^{3}$ Northeastern University Bouve College of Health Sciences School of Pharmacy, Boston, MA, USA. ${ }^{4}$ Department of Preventive Medicine, Seoul National University College of Medicine, Seoul, Korea. ${ }^{5}$ Cancer Research Institute, Seoul National University, Seoul, Korea. ${ }^{6}$ Department of Innovative Medical Science, Seoul National University College of Medicine, Seoul, Korea. ${ }^{7}$ Department of Family Medicine, Seoul National University College of Medicine, Seoul, Korea. ${ }^{8}$ Institute of Environmental Medicine, Seoul National University Medical Research Center, Seoul, Korea. ${ }^{9}$ Institute of Health Policy and Management, Seoul National University Medical Research Center, Seoul, Korea. ${ }^{\circledR}$ email: jiyeob.choi@gmail.com 
The Cochrane library conducted a meta-analysis of the association of MHT with specific CVD outcomes in RCTs. They conducted subgroup analyses based on the timing of MHT after menopause (timing hypothesis), but the study did not consider other confounding factors ${ }^{16}$. Another meta-analysis of RCTs examining the timing hypothesis for MHT and CVD risk supported the importance of the timing of initiation of MHT, and concluded that MHT may have beneficial effects on mortality and CVD events in younger menopausal women ${ }^{17}$. The previous meta-analysis studies of RCTs were limited to the timing hypothesis, and did not include other confounding factors that could affect the association between MHT and CVD. The most recent study was a systematic review of individual studies on the effects of timing, routes of administration, duration, and dose of MHT on CVD. However, that review was based on the findings of observational studies, and did not conduct pooled analyses of results owing to the diversity of the studies ${ }^{18}$. In addition, a previous meta-analysis of RCTs reported that inconsistent findings between the study designs may be due to the differences in the characteristics of the study populations, methodologic limitations of observational studies, and lower event rates and shorter duration of treatments in RCTs ${ }^{19}$.

Therefore, it is necessary to consistently examine the results of RCTs and observational studies by conducting various subgroup analyses and by comparing the characteristics of the included study populations. The aim of this study was to assess the association between MHT and CVD outcomes, and to compare the results of RCTs and observational studies through a systematic review and meta-analysis of RCTs and observational studies, respectively.

\section{Methods}

Search strategy and selection criteria. A literature search was conducted according to each study design (RCTs and observational studies) using the following search terms: ("cardiovascular diseases" OR "cerebrovascular disorder" OR "all-cause death" OR "cardiovascular death" OR "death" OR "mortality") AND ("hormone replacement therapy" OR "hormone therapy" OR "menopausal hormone therapy" OR "postmenopause"). Detailed search terms can be found in Supplementary File 1. The PubMed and EMBASE databases were searched to identify relevant articles published between January 2000 and December 2019. In the Cochrane review, studies published before 2000 were assessed as having a higher risk of bias than articles published after $2000^{16}$. Thus, the current study included only studies published after 2000.

The study selection criteria for the RCTs were based on the Cochrane reviews by Boardman et al. ${ }^{16}$. The same criteria were applied to the observational studies. After removing duplicates, exclusion criteria were separately applied to the remaining RCTs and observational studies. Only original articles of human studies published in English were included. Studies that did not report about relevant exposure or outcomes, or included either an ineligible population (premenopausal women or cancer survivors) or a duplicated study population, or had ineligible data to conduct the meta-analysis were excluded. Studies with an insufficient follow-up duration of less than 6 months in the RCTs or those with an ineligible study design (cross-sectional design) were also excluded. Reference lists of the relevant studies were manually screened to include more articles in our meta-analysis.

Data extraction and quality assessment. Two authors (JHC and MJJ) participated in study selection and data extraction. Two other authors (JEK and JYC) checked and reviewed the data in two steps. We extracted the data as follows: (1) characteristics of the included studies and populations, including author, year of publication, study design, follow-up duration, sample size, ethnicity, age at baseline, and underlying diseases; (2) exposure, including initiation of MHT after menopause, regimen type [estrogen only (E only) or combined estrogen-progesterone (combined EP)], route of administration, and duration, and recency of MHT; (3) outcomes, including all-cause death, cardiovascular death, stroke, venous thromboembolism (VTE), pulmonary embolism (PE), myocardial infarction (MI), coronary heart disease (CHD), angina, and revascularization; and (4) the effect estimates of the association between MHT and outcomes, such as hazard ratio, relative risk, odds ratio, 95\% confidence interval (CI), the number of exposed/non-exposed of MHT, and each event. Multivariable adjusted estimates were primarily extracted to reduce any confounding effects. If a study did not include the estimated values, the combined estimates were calculated based on the original estimates or the number of exposed/non-exposed, and each event was extracted as it was for the meta-analysis. Supplementary Tables S1-S4 provide details about the RCTs and observational studies included in the meta-analysis.

Representative studies were selected from one or more trials or studies, prioritizing the following selection criteria: (1) longest follow-up duration, (2) largest number of outcomes, or (3) largest number of participants. Detailed information on the selected representative RCTs and observational studies is presented in Supplementary Tables S5 and S6, respectively.

Quality assessment was conducted using the Jadad scale for RCTs, and the Newcastle-Ottawa Scale (NOS) for observational studies ${ }^{20,21}$. The Jadad scale consists of three domains: randomization ( $0-2$ points), blinding (0-2 points), and an account of all patients (0-1 point). We classified the quality of RCTs as good (4-5 points), fair ( 3 points), or poor ( $0-2$ points). The NOS is based on three domains: selection ( $0-4$ stars), comparability (0-2 stars), and exposure for cohort and nested case-control studies or outcomes for case-control studies (0-3 stars). The NOS, a star system, was converted to the Agency for Healthcare Research and Quality standards. The thresholds for assessing quality were as follows: (1) good: selection ( 3 or 4 stars) AND comparability ( 1 or 2 stars) AND outcome/exposure (2 or 3 stars); (2) fair: selection (2 stars) AND comparability (1 or 2 stars) AND outcome/exposure ( 2 or 3 stars); and (3) poor: selection (0 or 1 star) OR comparability (0 star) OR outcome/ exposure (0 or 1 star).

Statistical analysis. The association between MHT and each CVD outcome was evaluated using summary estimates (SE) and corresponding 95\% CIs. Heterogeneity among the included studies was assessed by the 

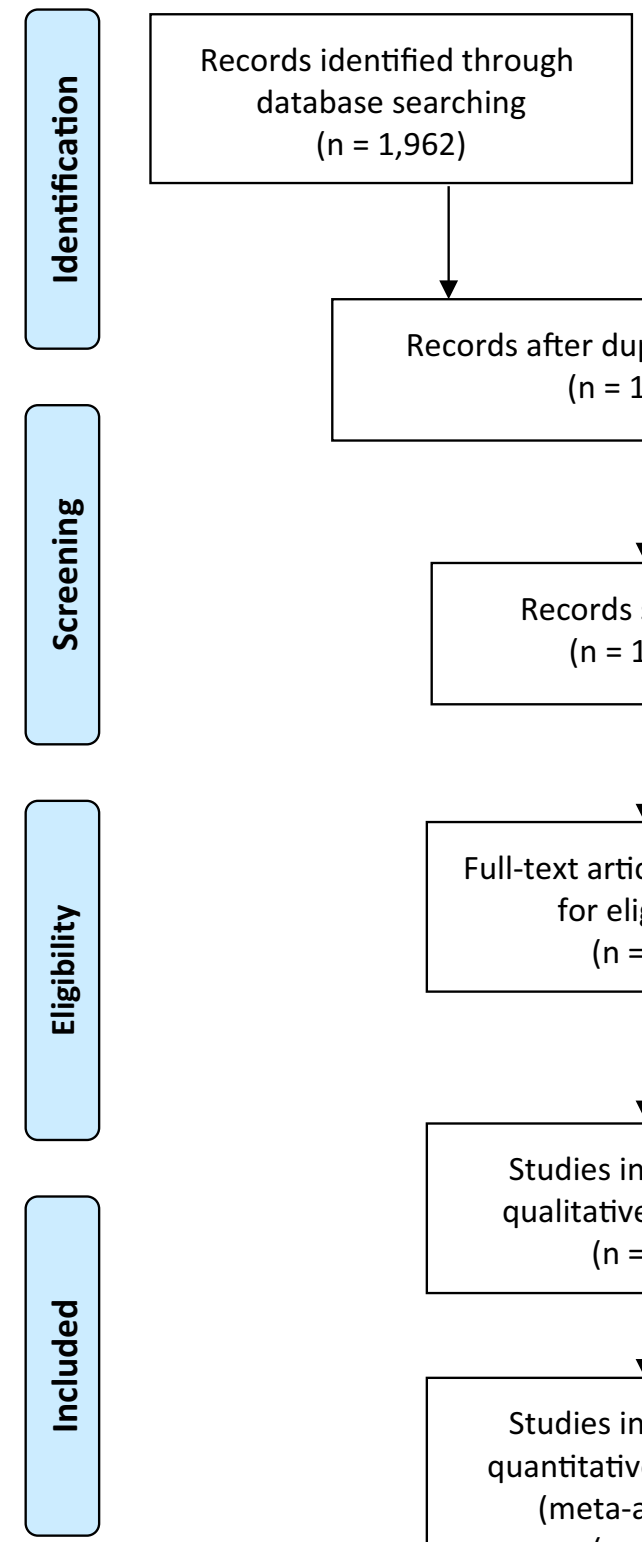
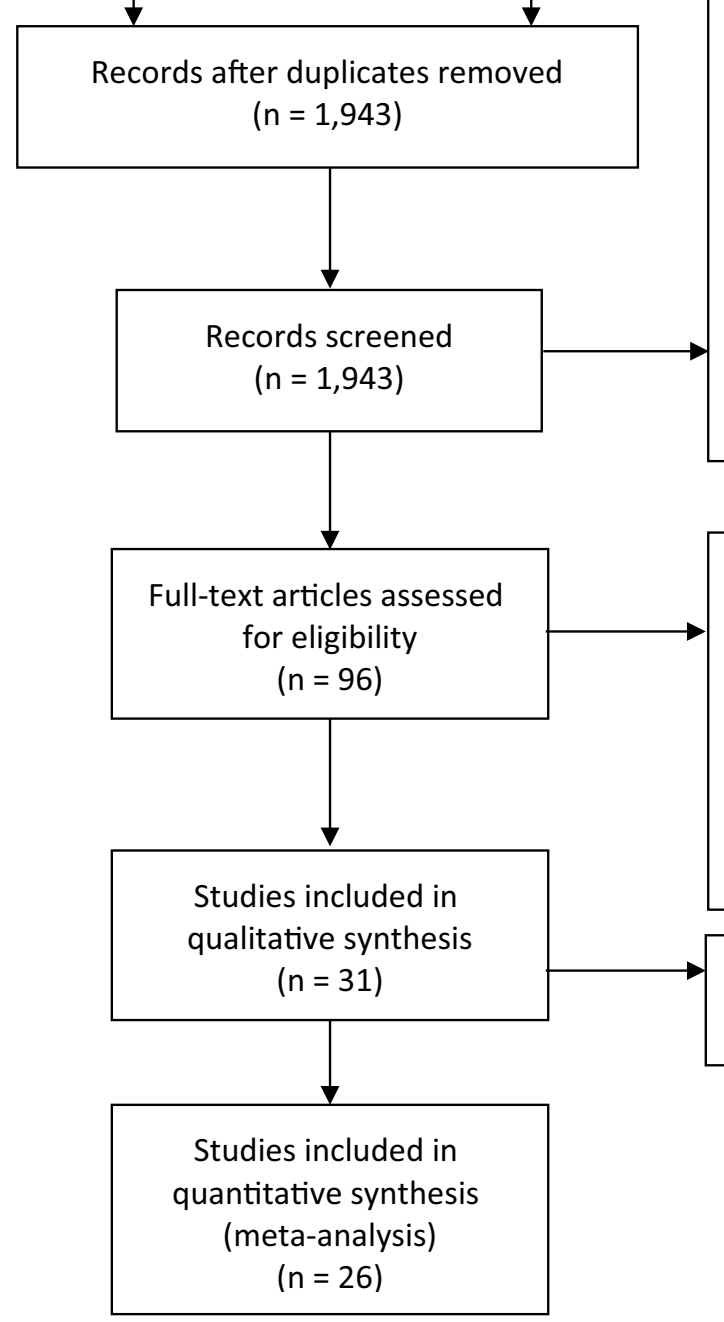

\section{1,847 records excluded}

1,075 not relevant topic 440 not original study

2 abstract only or not available article 200 not relevant outcome 76 not English article 44 not reporting MHT 10 not human study

65 full-text articles excluded

42 other subjects

11 duplicated study population

7 less than 6 months of follow-up

5 not a randomized controlled trial

5 studies did not provide eligible data for analysis

Figure 1. PRISMA flow diagram for study selection of the randomized controlled trials. From: Moher et al. ${ }^{48}$.

$\mathrm{I}^{2}$ index and Q statistics. We employed a fixed-effects model if the $\mathrm{I}^{2}$ was $<30 \%$ and the $P$-value by Q statistic was $>0.05$. If not, a random-effects model was used. In both RCTs and observational studies, we conducted subgroup analyses by regimen type (E only and combined EP), duration of MHT ( $<5$ and $\geq 5$ years), timing of initiation of MHT (early: age $<60$ years or initiation within 10 years since menopause; late: age $\geq 60$ years or initiation after 10 years since menopause), and underlying diseases (with or without). Subgroup analyses of observational studies were conducted by route of administration (oral and non-oral), study design (cohort, nested case-control, and case-control), recency of MHT (past and current), and study quality (good/fair and poor). We defined the timing of initiation of MHT based on the criteria of the timing hypothesis presented in a previous RCT meta-analysis conducted by Boardman et al. ${ }^{16}$ and Nudy et al. ${ }^{17}$. Subgroup analyses were performed when the number of studies was adequate. We evaluated the publication bias of included studies using symmetry funnel plots and Egger's test. Statistical analysis was performed using the "meta" packages in the R version 3.4.1 software (R Foundation for Statistical Computing).

\section{Results}

Eligible studies and characteristics. A total of 26 RCT studies (20 trials) and 47 observational studies were included in the final meta-analysis (Figs. 1, 2). We compared the characteristics of the included studies (Table 1). Most of the RCTs and observational studies were conducted in Europe or North America, and only a few studies were conducted in Asia. Eighteen RCTs (69.2\%) were published before 2006, the year in which the 

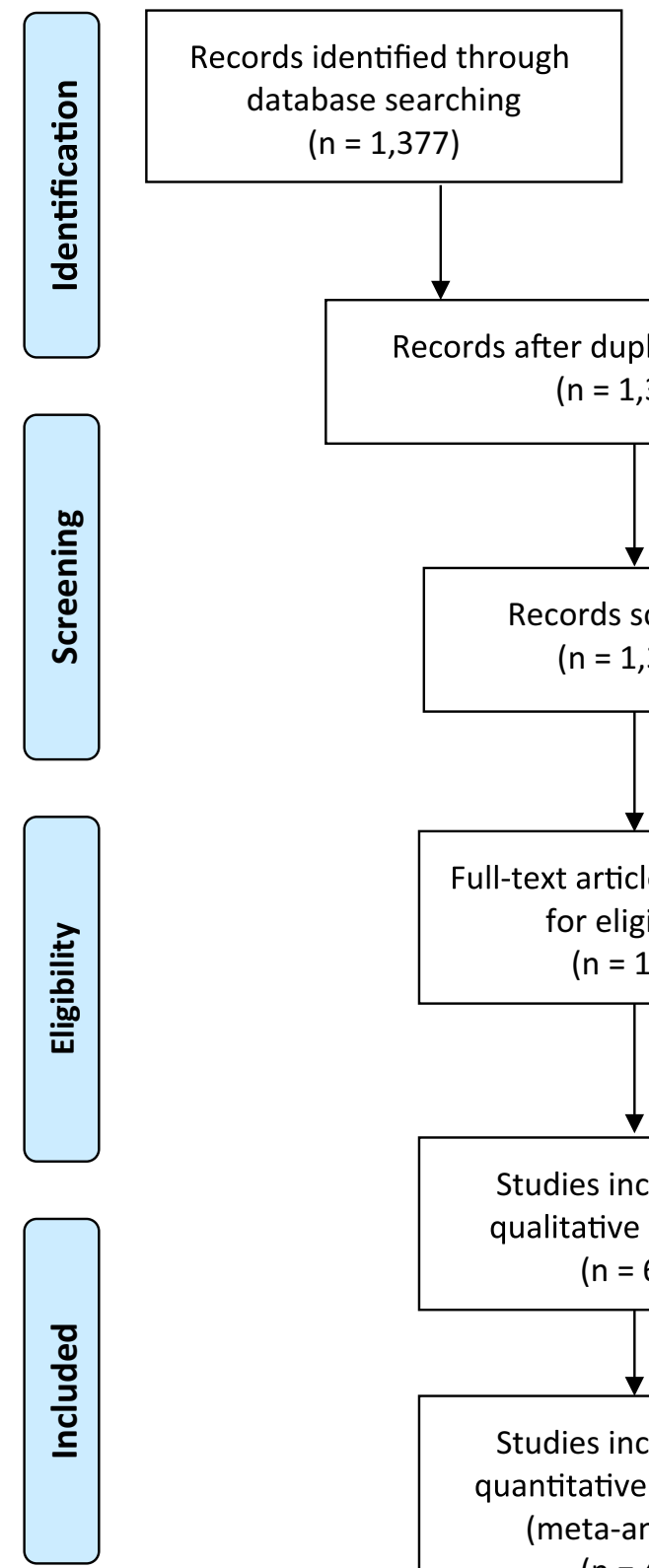

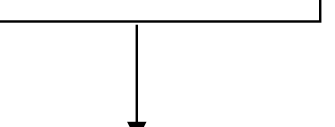

$(n=1,377)$

\section{Additional records identified}

through other sources

$(n=4)$
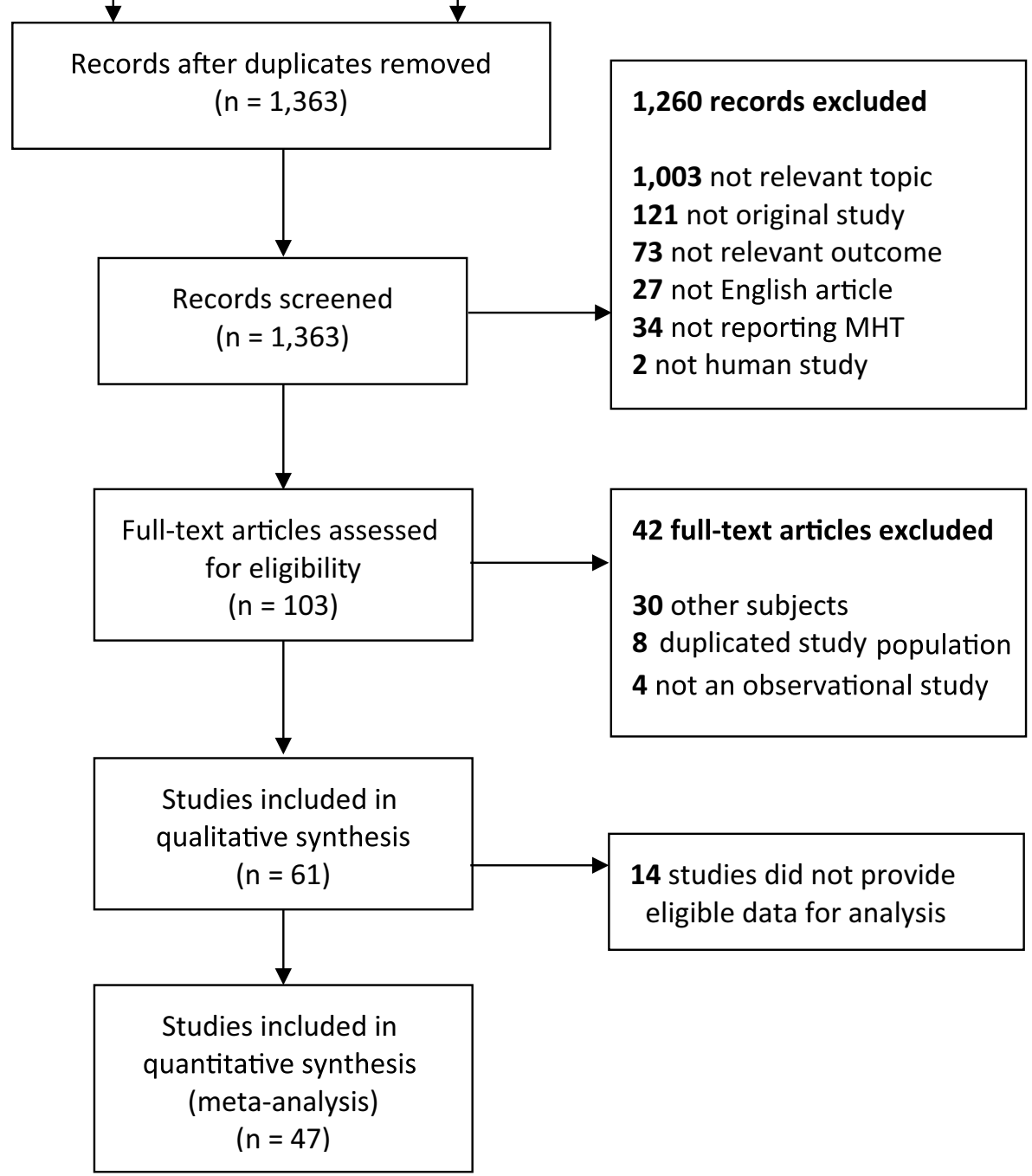

Figure 2. PRISMA flow diagram for study selection of the observational studies. From: Moher et al. ${ }^{48}$.

WHI findings were being actively reevaluated. Twenty-nine of the observational studies (61.7\%) were published after 2006. Study populations included in the RCTs were older than those included in the observational studies (median age, 63.6 vs. 60.6 years, respectively), and had more underlying diseases at baseline; subjects in the observational studies were relatively healthy. The route of MHT administration was oral in most RCTs, whereas some non-oral routes, such as transdermal or vaginal were used in the observational studies. The median followup duration of the RCTs was shorter than that of the observational studies ( 3.4 vs. 6.8 years). The observational studies included 30 cohort studies, 5 nested case-control studies, and 13 case-control studies.

Quality assessment. Most of the RCTs were classified as good or fair quality studies according to the Jadad scale. Among the 20 trials, 15 were good quality and 5 were fair quality studies (Supplementary Table S7). The RCTs were assessed as fair quality studies for the following reasons: (1) incomplete blinding that affected the results or (2) allocation based on laboratory tests that could have increased selection bias.

Results of the quality assessment of cohort, nested case-control, and case-control studies by the NOS can be found in Supplementary Tables S8-S10. Among the 30 cohort studies, 25 and 5 studies were assessed as good/ fair and poor quality studies, respectively. All 5 nested case-control studies were assessed as good quality. Studies with a cohort design were assessed as fair quality studies for the following reasons: (1) nurses or teachers included in the study population, increasing the risk of selection bias, or (2) MHT ascertained using a self-reported 


\begin{tabular}{|c|c|c|}
\hline & Randomized controlled trials ( $n=26$ including 20 trials) & Observational studies $(n=47)$ \\
\hline & $\mathrm{N}(\%)$ & $\mathbf{N}(\%)$ \\
\hline \multicolumn{3}{|l|}{ Ethnic group } \\
\hline Europe & $7(26.9)$ & $29(61.7)$ \\
\hline North America & $19(73.1)$ & $12(25.5)$ \\
\hline Rest of world & - & $6(12.8)$ \\
\hline \multicolumn{3}{|l|}{ Publication year } \\
\hline$\leq 2006$ & $18(69.2)$ & $18(38.3)$ \\
\hline$>2006$ & $8(30.8)$ & $29(61.7)$ \\
\hline Age at baseline, years (median, range) & $63.6(49.7-75.0)$ & $60.6(48.8-77.0)$ \\
\hline \multicolumn{3}{|l|}{ Timing of initiation of MHT $^{\star, \dagger}$} \\
\hline Reported & $22(84.6)$ & $36(76.6)$ \\
\hline Unreported & $4(15.4)$ & $11(23.4)$ \\
\hline \multicolumn{3}{|l|}{\begin{tabular}{|l|} 
Underlying diseases \\
\end{tabular}} \\
\hline With & $17(65.4)$ & $6(12.8)$ \\
\hline Without & $9(34.6)$ & $41(87.2)$ \\
\hline \multicolumn{3}{|l|}{ Regimen type $^{\star}$} \\
\hline Reported & $19(73.1)$ & $29(617)$ \\
\hline Unreported & $7(26.9)$ & $18(38.3)$ \\
\hline \multicolumn{3}{|l|}{ Route of administration ${ }^{*}$} \\
\hline Reported & $25(96.2)$ & $21(44.7)$ \\
\hline Unreported & $1(3.8)$ & $26(55.3)$ \\
\hline \multicolumn{3}{|l|}{ Duration of MHT ${ }^{*}$} \\
\hline Reported & $26(100.0)$ & $12(25.5)$ \\
\hline Unreported & - & $35(74.5)$ \\
\hline \multicolumn{3}{|l|}{ Outcomes $^{*}$} \\
\hline All-cause death & $16(61.5)$ & $15(31.9)$ \\
\hline Cardiovascular death & $10(38.5)$ & $6(12.8)$ \\
\hline Stroke & 12() & $14(29.8)$ \\
\hline VTE & $15(57.7)$ & $13(27.7)$ \\
\hline $\mathrm{PE}$ & $7(26.9)$ & $4(8.5)$ \\
\hline MI & $16(61.5)$ & $10(21.3)$ \\
\hline CHD & $4(15.4)$ & $7(14.9)$ \\
\hline Angina & $8(30.8)$ & $1(2.1)$ \\
\hline Revascularization & 7() & $0(0.0)$ \\
\hline Follow-up, years (median, range) & $3.4(0.7-18)$ & $6.8(1-21.5)$ \\
\hline \multicolumn{3}{|l|}{ Study type $^{*}$} \\
\hline Cohort study & - & $30(63.8)$ \\
\hline Case-control study & - & $13(27.7)$ \\
\hline Nested case-control study & - & $5(10.6)$ \\
\hline
\end{tabular}

Table 1. Overview of the characteristics of the included studies. CHD, coronary heart disease; MHT, menopausal hormone therapy; MI, myocardial infarction; PE, pulmonary embolism; VTE, venous thromboembolism. ${ }^{*}$ Individual studies often included more than one subgroup; †Women under 60 years of age or those in whom MHT was initiated within 10 years after menopause were included in the early subgroup; the others were included in the late subgroup.

questionnaire. Studies with a cohort design were assessed as poor quality studies for the following reasons: (1) no control for confounding factors, such as age, disease history, and other lifestyle factors; (2) outcomes ascertained using a self-reported questionnaire; or (3) follow-up duration insufficient or follow-up rate not reported. Among the 13 case-control studies, 7 studies were assessed as good quality, and 6 studies were assessed as poor quality. Studies with a case-control design were assessed as poor quality studies for the following reasons: (1) no control for confounding factors, such as age, disease history, and other lifestyle factors; (2) MHT ascertained via interview and interviewer not blinded to case/control status; or (3) response rates differed between cases and controls.

Meta-analysis of RCTs and observational studies. All-cause death and cardiovascular death. MHT was not associated with all-cause death (SE: 1.00, 95\% CI: 0.96-1.04 in RCTs; SE: 0.90, 95\% CI: 0.79-1.02 in observational studies) and cardiovascular death (SE: 0.96, 95\% CI: 0.83-1.12 in RCTs; SE: 0.81, 95\% CI: 0.61-1.07 in observational studies) in the pooled analysis of both RCTs and observational studies (Table 2). Subgroup 


\begin{tabular}{|c|c|c|c|c|c|c|c|c|}
\hline \multirow[b]{2}{*}{ Outcomes } & \multicolumn{4}{|c|}{ Randomized controlled trials } & \multicolumn{4}{|c|}{ Observational studies } \\
\hline & No. of trials & $\begin{array}{l}\text { Summary estimates }(95 \% \\
\text { CI })^{*}\end{array}$ & $I^{2}(\%)$ & P for heterogeneity & No. of studies & $\begin{array}{l}\text { Summary estimates }(95 \% \\
\text { CI })^{*}\end{array}$ & $\mathrm{I}^{2}(\%)$ & P for heterogeneity \\
\hline All-cause death & 17 & $1.00(0.96-1.04)$ & 0.0 & 0.61 & 15 & $0.90(0.79-1.02)$ & 88.7 & $<0.01$ \\
\hline Cardiovascular death & 11 & $0.96(0.83-1.12)$ & 39.5 & 0.09 & 6 & $0.81(0.61-1.07)$ & 32.9 & 0.19 \\
\hline Stroke & 13 & $1.14(1.04-1.25)$ & 0.0 & 0.98 & 13 & $0.98(0.85-1.13)$ & 71.4 & $<0.01$ \\
\hline VTE & 15 & $1.70(1.33-2.16)$ & 2.0 & 0.43 & 12 & $1.32(1.13-1.54)$ & 63.0 & $<0.01$ \\
\hline $\mathrm{PE}$ & 8 & $1.26(1.06-1.50)$ & 20.7 & 0.27 & 4 & $1.44(1.17-.1 .76)$ & 0.0 & 0.77 \\
\hline MI & 17 & $1.04(0.94-1.14)$ & 0.0 & 0.51 & 10 & $0.79(0.75-0.84)$ & 0.0 & 0.89 \\
\hline CHD & 5 & $1.02(0.94-1.10)$ & 0.0 & 0.43 & 7 & $0.91(0.72-1.15)$ & 75.4 & $<0.01$ \\
\hline Angina & 8 & $0.95(0.84-1.08)$ & 14.3 & 0.32 & 1 & $1.11(0.86-1.43)$ & - & - \\
\hline Revascularization & 7 & $0.96(0.87-1.06)$ & 14.1 & 0.32 & 0 & - & - & - \\
\hline
\end{tabular}

Table 2. Meta-analysis of randomized controlled trials and observational studies for menopausal hormonal therapy (MHT) and cardiovascular disease (CVD) outcomes. CHD, coronary heart disease; CI, confidence interval; MI, myocardial infarction; PE, pulmonary embolism; VTE, venous thromboembolism. * Summary estimates $(95 \% \mathrm{CI})$ were measured by fixed-effect models if $\mathrm{I}^{2}$ was $<30 \%$ and $\mathrm{P}$ for heterogeneity was $>0.05$; otherwise, the summary estimates $(95 \% \mathrm{CI})$ were measured by random-effect models.

analyses of RCTs did not identify any association between MHT and death (Table 3). In subgroup analyses of the observational studies, a decreased risk of all-cause death was observed among E only (SE: 0.85, 95\% CI: $0.77-0.95$ ) and early users after menopause (SE: 0.68, 95\% CI: 0.51-0.92; Table 4).

Stroke. MHT was associated with an increased risk of stroke in the pooled analysis of RCTs (SE: 1.14, 95\% CI: 1.04-1.25), although this was not observed in the pooled analysis of observational studies (SE: $0.98,95 \%$ CI:0.85-1.13; Table 2). In the subgroup analyses of RCTs, an increased risk of stroke was observed in combined EP users (SE: 1.14, 95\% CI: 1.01-1.29), users with a MHT duration $\geq 5$ years (SE: $1.13,95 \%$ CI: $1.03-1.25$ ), late users after menopause (SE: 1.17, 95\% CI: 1.01-1.37), and in women with an underlying disease at baseline (SE: 1.14, 95\% CI: 1.04-1.26; Table 3). In subgroup analysis of the observational studies, an increased risk of stroke was observed in women administered oral MHT (SE: 1.24, 95\% CI: 1.11-1.39), whereas a decreased risk of stroke was observed in women administered non-oral MHT (SE: 0.86, 95\% CI: 0.77-0.96). There was no difference in risk by duration of MHT (SE: 1.11, 95\% CI: 1.04-1.18 for < 5 years duration; SE: 1.22, 95\% CI: 1.16-1.29 for $\geq 5$ years duration; Table 4 ).

Venous thromboembolism. MHT was associated with an increased risk of VTE in the pooled results of both RCTs (SE: 1.70, 95\% CI: 1.33-2.16) and observational studies (SE: 1.32, 95\% CI: 1.13-1.54; Table 2). An increased risk of VTE was observed in combined EP users in both RCTs (SE: 2.28, 95\% CI: 1.64-3.18; Table 3) and observational studies (SE: 2.21, 95\% CI: 1.51-3.22; Table 4). This increased risk was also observed in late users after menopause (SE: $1.79,95 \%$ CI: 1.39-2.29) and women with an underlying disease in the RCTs (SE: $1.67,95 \%$ CI: 1.29-2.17; Table 3). It was not possible to evaluate the effects of underlying diseases on risk estimates in the observational studies, because women included in the observational studies were relatively healthy. Unlike findings from RCTs, an increased risk of VTE was observed in early users after menopause (SE: $1.55,95 \%$ CI: 1.26-1.92), and in women administered oral MHT (SE: 1.41, 95\% CI: 1.19-1.67; Table 4). There was no difference in risk by duration of MHT (SE: 1.93, 95\% CI: 1.10-3.36 for < 5 years duration; SE: 1.65, 95\% CI: 1.26-2.15 for $\geq 5$ years duration) in the RCTs, although an increased risk was observed in use of MHT for $<5$ years in observational studies (SE: 1.23, 95\% CI: 1.02-1.47; Tables 3, 4). Regardless of study quality, an increased risk of VTE was observed in the observational studies (SE: 1.28, 95\% CI: 1.08-1.51 in good and fair quality, SE: 1.60, 95\% CI: $1.15-2.22$ in poor quality; Table 4 ).

Pulmonary embolism. MHT was associated with an increased risk of PE in the pooled results of both RCTs (SE: 1.26, 95\% CI: 1.06-1.50) and observational studies (SE: 1.44, 95\% CI: 1.17-1.76; Table 2). In the subgroup analyses of RCTs, an increased risk of PE was observed in users with a MHT duration $\geq 5$ years (SE: $1.25,95 \%$ CI: 1.05-1.48), late users (SE: 1.88, 95\% CI: 1.28-2.78), and women with an underlying disease at baseline (SE: 1.24, 95\% CI: 1.05-1.48; Table 3).

Myocardial infarction and other outcomes. MHT was not associated with MI in the pooled results of RCTs (SE: 1.04, 95\% CI: 0.94-1.14), whereas a decreased risk of MI was observed in the pooled results of observational studies (SE: 0.79, 95\% CI: 0.75-0.84; Table 2). Subgroup analyses of RCTs did not reveal any association between MHT and MI (Table 3), whereas that of observational studies revealed a decreased risk in users with a MHT duration $\geq 5$ years (SE: $0.51,95 \%$ CI: $0.34-0.76$ ), and with a non-oral route of MHT administration (SE: 0.75 , 95\% CI: 0.60-0.93). A decreased risk of MI was observed regardless of regimen type, timing of initiation, underlying diseases, study design, and quality of observational studies (Table 4).

The pooled results from both RCTs and observational studies did not reveal any association between MHT and CHD (SE: 1.02, 95\% CI: 0.94-1.10 in RCTs; SE: 0.91, 95\% CI: 0.72-1.15 in observational studies; Table 2). 


\begin{tabular}{|c|c|c|c|c|c|}
\hline Variable & Subgroup & No. of trials & Summary estimates $(95 \% \mathrm{CI})^{*}$ & $\mathrm{I}^{2}(\%)$ & P for heterogeneity \\
\hline \multicolumn{6}{|l|}{ All-cause death } \\
\hline \multirow{2}{*}{ Regimen } & E only & 5 & $0.96(0.90-1.03)$ & 0.0 & 0.42 \\
\hline & Combined EP & 8 & $1.03(0.97-1.08)$ & 0.0 & 0.89 \\
\hline \multirow{2}{*}{ Duration } & $<5$ years & 12 & $1.10(0.94-1.29)$ & 0.0 & 0.92 \\
\hline & $\geq 5$ years & 5 & $0.99(0.91-1.07)$ & 43.2 & 0.13 \\
\hline \multirow{2}{*}{ Timing of initiation } & Early & 4 & $0.78(0.57-1.07)$ & 0.0 & 0.76 \\
\hline & Late & 15 & $1.00(0.96-1.05)$ & 0.0 & 0.62 \\
\hline \multirow{2}{*}{ Underlying diseases } & With & 11 & $1.00(0.96-1.04)$ & 0.2 & 0.44 \\
\hline & Without & 6 & $0.76(0.51-1.15)$ & 0.0 & 0.82 \\
\hline \multicolumn{6}{|l|}{ Cardiovascular death } \\
\hline \multirow{2}{*}{ Regimen } & E only & 4 & $0.96(0.85-1.07)$ & 0.0 & 0.59 \\
\hline & Combined EP & 4 & $1.04(0.94-1.15)$ & 0.0 & 0.64 \\
\hline \multirow{2}{*}{ Duration } & $<5$ years & 7 & $0.83(0.56-1.22)$ & 0.0 & 0.47 \\
\hline & $\geq 5$ years & 4 & $0.98(0.83-1.16)$ & 70.2 & 0.02 \\
\hline \multirow{2}{*}{ Timing of initiation } & Early & 1 & $0.26(0.11-0.64)$ & - & - \\
\hline & Late & 10 & $1.01(0.93-1.08)$ & 0.0 & 0.55 \\
\hline \multirow{2}{*}{ Underlying diseases } & With & 10 & $1.01(0.93-1.08)$ & 0.0 & 0.55 \\
\hline & Without & 1 & $0.26(0.11-0.64)$ & - & - \\
\hline \multicolumn{6}{|l|}{ Stroke } \\
\hline \multirow{2}{*}{ Regimen } & E only & 4 & $1.15(0.98-1.34)$ & 0.0 & 0.86 \\
\hline & Combined EP & 7 & $1.14(1.01-1.29)$ & 0.0 & 0.95 \\
\hline \multirow{2}{*}{ Duration } & $<5$ years & 9 & $1.21(0.91-1.63)$ & $\mid 0.0$ & 0.92 \\
\hline & $\geq 5$ years & 4 & $1.13(1.03-1.25)$ & 0.0 & 0.84 \\
\hline \multirow{2}{*}{ Timing of initiation } & Early & 5 & $1.33(0.91-1.93)$ & 0.0 & 0.57 \\
\hline & Late & 10 & $1.17(1.01-1.37)$ & 0.0 & 0.89 \\
\hline \multirow{2}{*}{ Underlying diseases } & With & 9 & $1.14(1.04-1.26)$ & 0.0 & 0.94 \\
\hline & Without & 4 & $1.05(0.63-1.78)$ & $\mid 0.0$ & 0.72 \\
\hline \multicolumn{6}{|l|}{ VTE } \\
\hline \multirow{2}{*}{ Regimen } & E only & 4 & $1.33(0.89-1.99)$ & 0.0 & 0.55 \\
\hline & Combined EP & 7 & $2.28(1.64-3.18)$ & 0.0 & 0.59 \\
\hline \multirow{2}{*}{ Duration } & $<5$ years & 10 & $1.93(1.10-3.36)$ & 0.0 & 0.49 \\
\hline & $\geq 5$ years & 5 & $1.65(1.26-2.15)$ & 27.7 & 0.24 \\
\hline Timing of initiation & Early & 2 & $0.69(0.25-1.93)$ & 0.0 & 0.65 \\
\hline Imining on intituation & Late & 13 & $1.79(1.39-2.29)$ & 0.0 & 0.53 \\
\hline UTderlving dicops & With & 9 & $1.67(1.29-2.17)$ & 0.0 & 0.75 \\
\hline Underlying diseases & Without & 6 & $1.87(0.71-4.94)$ & 45.3 & 0.10 \\
\hline PE & & & & & \\
\hline Rerimen & E only & 3 & $1.14(0.88-1.49)$ & 0.0 & 0.97 \\
\hline Regimen & Combined EP & 3 & $2.09(0.93-4.70)$ & 64.8 & 0.06 \\
\hline Durntion & $<5$ years & 3 & $1.89(0.72-4.92)$ & 23.8 & 0.27 \\
\hline Duration & $\geq 5$ years & 5 & $1.25(1.05-1.48)$ & 27.3 & 0.24 \\
\hline Timin of initiotion & Early & 3 & $1.73(0.87-3.42)$ & 14.0 & 0.31 \\
\hline Timing of initiation & Late & 7 & $1.88(1.28-2.78)$ & 0.0 & 0.49 \\
\hline UTdelving diceose & With & 5 & $1.24(1.05-1.48)$ & 0.0 & 0.47 \\
\hline Underlying diseases & Without & 3 & $2.08(0.34-12.59)$ & 50.4 & 0.13 \\
\hline MI & & & & & \\
\hline Rerimen & E only & 4 & $1.02(0.87-1.19)$ & 0.0 & 0.97 \\
\hline Regimen & Combined EP & 7 & $1.06(0.94-1.20)$ & 6.9 & 0.38 \\
\hline Durntion & $<5$ years & 12 & $1.03(0.69-1.55)$ & 0.0 & 0.62 \\
\hline Duration & $\geq 5$ years & 5 & $1.02(0.89-1.17)$ & 34.4 & 0.19 \\
\hline Timing of initintion & Early & 5 & $0.74(0.50-1.11)$ & 0.0 & 0.50 \\
\hline Timing of initiation & Late & 14 & $1.00(0.86-1.17)$ & 0.0 & 0.66 \\
\hline Und relving disese & With & 10 & $1.04(0.94-1.14)$ & 0.0 & 0.70 \\
\hline Underlying diseases & Without & 7 & $1.00(0.43-2.29)$ & 30.7 & 0.19 \\
\hline CHD & & & & & \\
\hline Continued & & & & & \\
\hline
\end{tabular}




\begin{tabular}{|c|c|c|c|c|c|}
\hline Variable & Subgroup & No. of trials & Summary estimates $(95 \% \mathrm{CI})^{*}$ & $\mathrm{I}^{2}(\%)$ & P for heterogeneity \\
\hline \multirow{2}{*}{ Regimen } & E only & 2 & $0.93(0.81-1.07)$ & 0.0 & 0.83 \\
\hline & Combined EP & 4 & $1.05(0.96-1.15)$ & 0.0 & 0.51 \\
\hline \multirow{2}{*}{ Duration } & $<5$ years & 2 & $1.02(0.80-1.30)$ & 33.0 & 0.22 \\
\hline & $\geq 5$ years & 3 & $1.01(0.93-1.10)$ & 13.0 & 0.32 \\
\hline \multirow{2}{*}{ Timing of initiation } & Early & 3 & $0.94(0.66-1.33)$ & 44.0 & 0.17 \\
\hline & Late & 4 & $1.00(0.87-1.14)$ & 0.0 & 0.66 \\
\hline \multirow{2}{*}{ Underlying diseases } & With & 4 & $1.01(0.93-1.09)$ & 1.7 & 0.38 \\
\hline & Without & 1 & $1.12(0.90-1.40)$ & - & - \\
\hline \multicolumn{6}{|l|}{ Angina } \\
\hline \multirow{2}{*}{ Regimen } & E only & 2 & $0.96(0.78-1.17)$ & 0.0 & 0.69 \\
\hline & Combined EP & 4 & $0.85(0.71-1.01)$ & 0.0 & 0.48 \\
\hline \multirow{2}{*}{ Duration } & $<5$ years & 4 & $1.12(0.76-1.63)$ & 32.6 & 0.22 \\
\hline & $\geq 5$ years & 4 & $0.91(0.79-1.05)$ & 0.0 & 0.57 \\
\hline \multirow{2}{*}{ Timing of initiation } & Early & 1 & $0.87(0.54-1.41)$ & - & - \\
\hline & Late & 7 & $1.00(0.86-1.17)$ & 28.3 & 0.21 \\
\hline \multirow{2}{*}{ Underlying diseases } & With & 6 & $0.94(0.83-1.07)$ & 5.1 & 0.38 \\
\hline & Without & 2 & $5.90(0.71-49.13)$ & 0.0 & 0.87 \\
\hline \multicolumn{6}{|l|}{ Revascularization } \\
\hline \multirow{2}{*}{ Regimen } & E only & 3 & $0.91(0.78-1.07)$ & 0.0 & 0.75 \\
\hline & Combined EP & 3 & $1.00(0.87-1.15)$ & 0.0 & 0.79 \\
\hline \multirow{2}{*}{ Duration } & $<5$ years & 4 & $0.90(0.54-1.49)$ & 43.7 & 0.15 \\
\hline & $\geq 5$ years & 3 & $0.98(0.88-1.09)$ & 0.0 & 0.73 \\
\hline \multirow{2}{*}{ Timing of initiation } & Early & 2 & $0.78(0.54-1.13)$ & 0.0 & 0.67 \\
\hline & Late & 6 & $0.97(0.86-1.10)$ & 21.8 & 0.27 \\
\hline \multirow{2}{*}{ Underlying diseases } & With & 6 & $0.96(0.87-1.07)$ & 25.3 & 0.24 \\
\hline & Without & 1 & $0.50(0.05-5.43)$ & - & \\
\hline
\end{tabular}

Table 3. Subgroup analyses for menopausal hormonal therapy (MHT) and cardiovascular disease (CVD) outcomes in randomized controlled trials. CHD, coronary heart disease; CI, confidence interval; MI, myocardial infarction; E, estrogen; EP, estrogen and progesterone; PE, pulmonary embolism; VTE, venous thromboembolism. ${ }^{\star}$ Summary estimates $(95 \% \mathrm{CI})$ were measured by fixed-effect models if the $\mathrm{I}^{2}$ was $<30 \%$ and $\mathrm{P}$ for heterogeneity was $>0.05$; otherwise, the summary estimates $(95 \% \mathrm{CI})$ were measured by randomeffect models.

In the pooled results of the RCTs, there was also no association between MHT and revascularization (SE: 0.96, 95\% CI: 0.87-1.06), or angina (SE: 0.95, 95\% CI: 0.84-1.08; Table 2).

The forest plots for all analyses can be found in Supplementary Figures S1-S4.

Publication bias. There was no evidence of publication bias for all-cause death, cardiovascular death, stroke, VTE, PE, MI, CHD, angina, or revascularization in the RCTs or the observational studies (Egger's $P$-value $>0.05$ ). The funnel plots and Egger's $P$-values calculated for the assessment of publication bias are included in Supplementary Figures S1 and S2.

\section{Discussion}

Summary of findings. RCTs had a shorter follow-up duration than did observational studies, and the study populations in the RCTs were older, initiated MHT late after menopause, and had more underlying diseases than those in the observational studies. RCTs and observational studies both showed that MHT was associated with an increased risk of VTE and PE, although only the RCTs revealed an increased risk of stroke among those administered MHT. A decreased risk of MI by MHT was identified in the observational studies, but the RCTs did not show this association. Although still unexplained in the current literature, differential clinical effects according to regimen type, timing of initiation, underlying disease, and route of administration were identified in subgroup analyses.

Comparison of findings with previous systematic reviews and a meta-analysis. Our meta-analysis of RCTs was based on the Cochrane review published in $2015^{16}$. Among the included RCTs, 13 trials overlapped with those included in the Cochrane review ${ }^{12,22-33}$. Four other trials (ESPRIT, HERS, WHI I, and WHI II) ${ }^{13,14,34-36}$ were included according to our inclusion criteria. Three trials (EMS, KEEPS, and PHASE) ${ }^{37-39}$ were newly identified in this study. Two other trials ${ }^{40,41}$ with a higher risk of bias than other studies, and one trial ${ }^{27}$ assessing recurrent VTE as the outcome, were excluded. 


\begin{tabular}{|c|c|c|c|c|c|}
\hline Variable & Subgroup & No. of studies & Summary estimates $(95 \% \mathrm{CI})^{*}$ & $I^{2}(\%)$ & P for heterogeneity \\
\hline \multicolumn{6}{|l|}{ All-cause death } \\
\hline \multirow{2}{*}{ Regimen } & E only & 7 & $0.85(0.77-0.95)$ & 59.5 & 0.02 \\
\hline & Combined EP & 7 & $0.61(0.34-1.09)$ & 99.3 & $<0.01$ \\
\hline \multirow{2}{*}{ Duration } & $<5$ years & 2 & $0.65(0.25-1.64)$ & 98.0 & $<0.01$ \\
\hline & $\geq 5$ years & 2 & $0.81(0.50-1.30)$ & 88.5 & $<0.01$ \\
\hline \multirow{2}{*}{ Timing of initiation } & Early & 8 & $0.68(0.51-0.92)$ & 94.6 & $<0.01$ \\
\hline & Late & 6 & $0.94(0.73-1.21)$ & 83.3 & $<0.01$ \\
\hline \multirow{2}{*}{ Routes of administration } & Oral & 2 & $1.01(0.94-1.08)$ & 0.0 & 0.75 \\
\hline & Non-oral & 3 & $0.83(0.65-1.07)$ & 49.1 & 0.14 \\
\hline \multirow{2}{*}{ Underlying diseases } & With & 3 & $1.26(0.34-4.64)$ & 91.6 & $<0.01$ \\
\hline & Without & 12 & $0.89(0.78-1.01)$ & 89.0 & $<0.01$ \\
\hline \multirow{2}{*}{ Recency of MHT } & Past & 4 & $0.95(0.86-1.04)$ & 57.8 & 0.07 \\
\hline & Current & 4 & $0.90(0.78-1.03)$ & 85.8 & $<0.01$ \\
\hline \multirow{3}{*}{ Study design } & Cohort & 15 & $0.90(0.79-1.02)$ & 88.7 & $<0.01$ \\
\hline & Case-control & 0 & - & - & - \\
\hline & Nested case-control & 0 & - & - & - \\
\hline \multirow{2}{*}{ Study quality } & Good and fair & 12 & $0.89(0.78-1.01)$ & 89.0 & $<0.01$ \\
\hline & Poor & 3 & $1.26(0.34-4.64)$ & 91.6 & $<0.01$ \\
\hline \multicolumn{6}{|l|}{ Stroke } \\
\hline \multirow{2}{*}{ Regimen } & E only & 9 & $1.02(0.90-1.16)$ & 65.9 & $<0.01$ \\
\hline & Combined EP & 6 & $1.05(0.81-1.35)$ & 92.0 & $<0.01$ \\
\hline \multirow{2}{*}{ Duration } & $<5$ years & 3 & $1.11(1.04-1.18)$ & 0.0 & 0.43 \\
\hline & $\geq 5$ years & 2 & $1.22(1.16-1.29)$ & 5.0 & 0.30 \\
\hline \multirow{2}{*}{ Timing of initiation } & Early & 4 & $0.81(0.62-1.06)$ & 22.0 & 0.28 \\
\hline & Late & 6 & $0.91(0.69-1.19)$ & 59.2 & 0.03 \\
\hline \multirow{2}{*}{ Routes of administration } & Oral & 5 & $1.24(1.11-1.39)$ & 50.7 & 0.09 \\
\hline & Non-oral & 5 & $0.86(0.77-0.96)$ & 0.0 & 0.91 \\
\hline \multirow{2}{*}{ Underlying diseases } & With & 3 & $1.19(0.27-5.26)$ & 81.8 & $<0.01$ \\
\hline & Without & 10 & $1.00(0.88-1.14)$ & 69.6 & $<0.01$ \\
\hline \multirow{2}{*}{ Recency of MHT } & Past & 3 & $1.03(0.99-1.07)$ & 0.0 & 0.99 \\
\hline & Current & 3 & $1.17(1.12-1.22)$ & 0.0 & 0.88 \\
\hline \multirow{3}{*}{ Study design } & Cohort & 9 & $0.97(0.82-1.15)$ & 55.1 & 0.02 \\
\hline & \begin{tabular}{|l|} 
Case-control \\
\end{tabular} & 2 & $0.84(0.75-0.94)$ & $\mid 0.0$ & 0.44 \\
\hline & Nested case-control & 2 & $1.22(1.11-1.34)$ & $\mid 0.0$ & 0.82 \\
\hline \multirow{2}{*}{ Study quality } & Good and fair & 10 & $0.99(0.87-1.14)$ & 72.1 & $<0.01$ \\
\hline & Poor & 3 & $1.27(0.40-4.02)$ & 77.3 & 0.01 \\
\hline VTE & & & & & \\
\hline Dorimat & E only & 8 & $0.93(0.79-1.08)$ & 0.0 & 0.51 \\
\hline Regimen & Combined EP & 6 & $2.21(1.51-3.22)$ & 90.1 & $<0.01$ \\
\hline Dumetion & $<5$ years & 4 & $1.23(1.02-1.47)$ & 0.0 & 0.88 \\
\hline Duration & $\geq 5$ years & 2 & $1.19(0.95-1.51)$ & 0.0 & 0.39 \\
\hline Timing finitiotion & Early & 6 & $1.55(1.26-1.92)$ & 31.4 & 0.20 \\
\hline Timing of initiation & Late & 5 & $1.27(0.87-1.86)$ & 2.9 & $<0.01$ \\
\hline Boutes of adminictration & Oral & 9 & $1.41(1.19-1.67)$ & 72.5 & $<0.01$ \\
\hline Routes of administration & Non-oral & 7 & $0.81(0.60-1.09)$ & 70.8 & $<0.01$ \\
\hline Underlving diceper & With & 0 & - & - & - \\
\hline Underlyıng diseases & Without & 12 & $1.32(1.13-1.54)$ & 63.0 & $<0.01$ \\
\hline D & Past & 6 & $1.07(0.97-1.19)$ & 31.4 & 0.20 \\
\hline Recency of MHT & Current & 6 & $1.52(1.45-1.60)$ & 0.0 & 0.66 \\
\hline & Cohort & 6 & $1.25(1.01-1.55)$ & 38.9 & 0.15 \\
\hline Study design & \begin{tabular}{|l|} 
Case-control \\
\end{tabular} & 5 & $1.43(1.07-1.91)$ & 80.8 & $<0.01$ \\
\hline & Nested case-control & 1 & $1.34(1.03-1.73)$ & - & - \\
\hline Studvoulity & Good and fair & 10 & $1.28(1.08-1.51)$ & 65.7 & $<0.01$ \\
\hline Study quality & Poor & 2 & $1.60(1.15-2.22)$ & 0.0 & 0.35 \\
\hline MI & & & & & \\
\hline Continued & & & & & \\
\hline
\end{tabular}




\begin{tabular}{|c|c|c|c|c|c|}
\hline Variable & Subgroup & No. of studies & Summary estimates $(95 \% \mathrm{CI})^{*}$ & $\mathrm{I}^{2}(\%)$ & P for heterogeneity \\
\hline \multirow{2}{*}{ Regimen } & E only & 9 & $0.85(0.79-0.91)$ & 0.0 & 0.67 \\
\hline & Combined EP & 8 & $0.77(0.71-0.84)$ & 20.4 & 0.27 \\
\hline \multirow{2}{*}{ Duration } & $<5$ years & 3 & $0.91(0.73-1.12)$ & 0.0 & 0.54 \\
\hline & $\geq 5$ years & 2 & $0.51(0.34-0.76)$ & 0.2 & 0.32 \\
\hline \multirow{2}{*}{ Timing of initiation } & Early & 3 & $0.78(0.62-0.98)$ & 0.0 & 0.80 \\
\hline & Late & 4 & $0.79(0.73-0.84)$ & 0.0 & 0.68 \\
\hline \multirow{2}{*}{ Routes of administration } & Oral & 2 & $0.87(0.57-1.32)$ & 83.3 & 0.01 \\
\hline & Non-oral & 3 & $0.75(0.60-0.93)$ & 0.0 & 0.45 \\
\hline \multirow{2}{*}{ Underlying diseases } & With & 1 & $0.84(0.72-0.98)$ & - & - \\
\hline & Without & 9 & $0.79(0.74-0.84)$ & 0.0 & 0.88 \\
\hline \multirow{2}{*}{ Recency of MHT } & Past & 4 & $0.84(0.75-0.95)$ & 0.0 & 0.68 \\
\hline & Current & 4 & $0.81(0.59-1.10)$ & 76.7 & $<0.01$ \\
\hline \multirow{3}{*}{ Study design } & Cohort & 5 & $0.85(0.76-0.95)$ & 0.0 & 0.79 \\
\hline & \begin{tabular}{|l|} 
Case-control \\
\end{tabular} & 5 & $0.77(0.72-0.83)$ & 0.0 & 0.94 \\
\hline & Nested case-control & 0 & - & - & - \\
\hline \multirow{2}{*}{ Study quality } & Good and fair & 7 & $0.78(0.73-0.84)$ & 0.0 & 0.78 \\
\hline & Poor & 3 & $0.84(0.74-0.95)$ & 0.0 & 0.89 \\
\hline
\end{tabular}

Table 4. Subgroup analyses for MHT and CVD outcomes in observational studies. CI, confidence interval; MI, myocardial infarction; E, estrogen; EP, estrogen and progesterone; VTE, venous thromboembolism. ${ }^{*}$ Summary estimates $\left(95 \% \mathrm{CI}\right.$ ) were measured by fixed-effect models if the $\mathrm{I}^{2}$ was $<30 \%$ and $\mathrm{P}$ for heterogeneity was $>0.05$; otherwise, the summary estimates $(95 \% \mathrm{CI})$ were measured by random-effect models.

Consistent with the Cochrane review ${ }^{16}$, our pooled results from the RCTs showed an increased risk of stroke, VTE, and PE among MHT users. However, the effect size in the current study was decreased compared to those in the Cochrane review. We considered multivariable adjusted-estimates as a priority for the meta-analysis, thus potentially attenuating the effects of confounding factors. Nudy et al. conducted another RCT-based meta-analysis to assess the assumption of the timing hypothesis ${ }^{17}$. They reported that younger MHT users had a decreased risk of all-cause death and cardiac events (a composite of cardiac mortality and non-fatal MI), whereas the risk of a composite of stroke, transient ischemic attack (TIA), and systemic embolism increased as age increased. Unlike the Cochrane review ${ }^{16}$ and our meta-analysis, they integrated stroke, TIA, and systemic embolism as an outcome. Thus, it is not possible to compare the results with those from this study.

Two previous RCT-based meta-analyses ${ }^{16,17}$ did not assess the effects by confounding factors owing to insufficient information, but a more recent systematic review ${ }^{18}$ reported the effects of the timing of initiation, route of administration, duration, and dose on CVD risk. They reported that a low dose of oral MHT and transdermal MHT may have beneficial effects on CVD, including stroke and VTE. However, they did not report a synthesis of results, and most of the results were derived from observational studies. They reviewed 33 studies that included 6 RCTs and 27 observational studies; thus, it is difficult to compare the results between RCTs and observational studies. As another limitation, they reported that most of the included studies had a low or moderate evidence level based on quality assessment. We conducted subgroup analysis according to the quality of the observational studies. Although we investigated the effect of MHT on CVD through a meta-analysis in a manner similar to older more conventional studies, our study is comparable to the most recent review.

Comparison of findings between RCTs and observational studies. Our pooled analysis of both RCTs and observational studies identified consistent findings with respect to thrombotic events, and inconsistent findings regarding stroke and MI. However, differential associations in the subgroup analyses were observed. In our meta-analysis, timing of initiation and underlying diseases at baseline were likely to affect CVD outcomes. Mostly, late users and women who had an underlying disease at baseline had an increased risk of CVD outcomes, whereas early users and relatively healthy women had a decreased risk in both RCTs and observational studies. We found that the route of MHT administration was a possible factor for differential associations with CVD outcomes. Oral MHT was related to an increased risk of thrombotic events and stroke, whereas transdermal and vaginal MHT were comparatively safer than oral MHT in a review of observational studies ${ }^{18}$; however, this information was not available for RCTs ${ }^{16,17}$. Our subgroup analyses of observational studies according to route of administration supported findings from the most recent review ${ }^{18,42}$. The recent Nurses' Health Study and the WHI Observational Study also have suggested the safety of low-dose vaginal estrogen with respect to the risk of CVD and cancer ${ }^{43,44}$.

Strengths and limitations. In the current study, we compared the characteristics of RCTs and observational studies, and identified possible reasons for inconsistent findings through various subgroup analyses. However, it is necessary to be cautious when interpreting our findings owing to some limitations. First, most of the study subjects were Europeans or North Americans. Thus, it was difficult to identify ethnic differences, for 
example, the prevalence of CVD, and age at natural menopause ${ }^{45,46}$. Second, some observational studies defined MHT through a self-reported questionnaire, and the included RCTs used different treatment regimens. Our subgroup analysis only considered E only and combined EP regimen types, and therefore, we were unable to assess the effect of more detailed regimens. Although the heterogeneity of the observational studies was higher than that of the RCTs, it was slightly attenuated in the subgroup analyses. Third, methodologic limitations for the control of confounding effects may remain. However, because we extracted multivariable adjusted-estimates for the associations as a priority, some of the confounders may have been controlled in this study. Although atrial fibrillation (AF) has been known to be strongly associated with thrombotic events, most of the included studies in our meta-analysis did not take this into account ${ }^{47}$. Therefore, the potential strong association between AF and thrombotic events may have contributed to the consistent findings of increased risk of thrombotic events in both RCTs and observational studies. Further studies are necessary to evaluate well-known risk factors, such as AF, to identify the association between MHT and risk of thrombotic events. Finally, the largest WHI trials may have contributed to the RCT findings. When we performed sensitivity analyses after excluding the WHI trials, the results did not change except for stroke, which suggested that the increased risk of stroke in the RCTs may be overestimated, or may reflect the characteristics of women who received MHT. Nevertheless, to the best of our knowledge, our study is the first meta-analysis of both RCTs and observational studies that takes into account as many factors as possible, unlike previous meta-analyses. The meta-analysis of observational studies can also be comparable to that of RCTs, as the observational studies often have longer follow-ups and are conducted in a more real-world setting. Although the included observational studies were rated 'good' or 'fair' in the quality assessment, healthy user bias may remain, and therefore, the results should be interpreted with caution.

\section{Conclusion}

Our findings support the idea that the risks and benefits of MHT are likely to depend on the characteristics of the women who are treated. MHT is still not recommended for the prevention of chronic diseases; however, it may have beneficial effects with respect to CVD and mortality in postmenopausal women with severe menopausal symptoms, after sufficient consideration of underlying diseases and timing of treatment initiation. Moreover, the use of non-oral types of MHT for menopausal symptoms may be suggested for women at high risk of VTE and stroke than oral types. Further studies to investigate the influence of ethnicity or specific MHT types are required.

Received: 27 March 2020; Accepted: 12 November 2020

Published online: 26 November 2020

\section{References}

1. Hayward, C. S., Kelly, R. P. \& Collins, P. The roles of gender, the menopause and hormone replacement on cardiovascular function. Cardiovasc. Res. 46, 28-49. https://doi.org/10.1016/s0008-6363(00)00005-5 (2000).

2. Wake, R. \& Yoshiyama, M. Gender differences in ischemic heart disease. Rec. Pat. Cardiovasc. Drug Discov. 4, 234-240 (2009).

3. Raz, L. Estrogen and cerebrovascular regulation in menopause. Mol. Cell Endocrinol. 389, 22-30. https://doi.org/10.1016/j. mce.2014.01.015 (2014).

4. Pu, D., Tan, R., Yu, Q. \& Wu, J. Metabolic syndrome in menopause and associated factors: a meta-analysis. Climacteric 20, 583-591. https://doi.org/10.1080/13697137.2017.1386649 (2017)

5. Anagnostis, P. et al. Menopausal hormone therapy and cardiovascular risk. Where are we now?. Curr. Vasc. Pharmacol. https:// doi.org/10.2174/1570161116666180709095348 (2018).

6. Iorga, A. et al. The protective role of estrogen and estrogen receptors in cardiovascular disease and the controversial use of estrogen therapy. Biol. Sex Differ. 8, 33. https://doi.org/10.1186/s13293-017-0152-8 (2017).

7. Wolf, P. H., Madans, J. H., Finucane, F. F., Higgins, M. \& Kleinman, J. C. Reduction of cardiovascular disease-related mortality among postmenopausal women who use hormones: evidence from a national cohort. Am. J. Obstet. Gynecol. 164, 489-494. https ://doi.org/10.1016/s0002-9378(11)80006-2 (1991).

8. Grodstein, F. et al. A prospective, observational study of postmenopausal hormone therapy and primary prevention of cardiovascular disease. Ann. Intern Med. 133, 933-941. https://doi.org/10.7326/0003-4819-133-12-200012190-00008 (2000).

9. Petitti, D. B., Sidney, S. \& Quesenberry, C. P. Jr. Hormone replacement therapy and the risk of myocardial infarction in women with coronary risk factors. Epidemiology 11, 603-606. https://doi.org/10.1097/00001648-200009000-00018 (2000).

10. Hulley, S. et al. Randomized trial of estrogen plus progestin for secondary prevention of coronary heart disease in postmenopausal women. Heart and Estrogen/progestin Replacement Study (HERS) Research Group. JAMA 280, 605-613. https://doi.org/10.1001/ jama.280.7.605 (1998).

11. Rossouw, J. E. et al. Risks and benefits of estrogen plus progestin in healthy postmenopausal women: principal results From the Women's Health Initiative randomized controlled trial. JAMA 288, 321-333. https://doi.org/10.1001/jama.288.3.321 (2002).

12. Viscoli, C. M. et al. A clinical trial of estrogen-replacement therapy after ischemic stroke. N. Engl. J. Med. 345, 1243-1249. https ://doi.org/10.1056/NEJMoa010534 (2001).

13. Manson, J. E. et al. Menopausal hormone therapy and long-term all-cause and cause-specific mortality: the women's health initiative randomized trials. JAMA 318, 927-938. https://doi.org/10.1001/jama.2017.11217 (2017).

14. Cherry, N., McNamee, R., Heagerty, A., Kitchener, H. \& Hannaford, P. Long-term safety of unopposed estrogen used by women surviving myocardial infarction: 14-year follow-up of the ESPRIT randomised controlled trial. BJOG 121, 700-705. https://doi. org/10.1111/1471-0528.12598 (2014) (discussion 705).

15. Chen, L., Mishra, G. D., Dobson, A. J., Wilson, L. F. \& Jones, M. A. Protective effect of hormone therapy among women with hysterectomy/oophorectomy. Hum. Reprod. 32, 885-892. https://doi.org/10.1093/humrep/dex017 (2017).

16. Boardman, H. M. et al. Hormone therapy for preventing cardiovascular disease in post-menopausal women. Cochrane Database Syst. Rev. https://doi.org/10.1002/14651858.CD002229.pub4 (2015).

17. Nudy, M., Chinchilli, V. M. \& Foy, A. J. A systematic review and meta-regression analysis to examine the "timing hypothesis" of hormone replacement therapy on mortality, coronary heart disease, and stroke. Int. J. Cardiol. Heart Vasc. 22, 123-131. https:// doi.org/10.1016/j.ijcha.2019.01.001 (2019).

18. Oliver-Williams, C. et al. The route of administration, timing, duration and dose of postmenopausal hormone therapy and cardiovascular outcomes in women: a systematic review. Hum. Reprod. Update 25, 257-271. https://doi.org/10.1093/humupd/dmy03 9 (2019). 
19. Yang, D., Li, J., Yuan, Z. \& Liu, X. Effect of hormone replacement therapy on cardiovascular outcomes: a meta-analysis of randomized controlled trials. PLoS ONE 8, e62329. https://doi.org/10.1371/journal.pone.0062329 (2013).

20. Clark, H. D. et al. Assessing the quality of randomized trials: reliability of the Jadad scale. Control Clin. Trials 20, 448-452 (1999).

21. Peterson, J., Welch, V., Losos, M. \& Tugwell, P. The Newcastle-Ottawa scale (NOS) for assessing the quality of nonrandomised studies in meta-analyses.

22. Schierbeck, L. L. et al. Effect of hormone replacement therapy on cardiovascular events in recently postmenopausal women: randomised trial. BMJ 345, e6409. https://doi.org/10.1136/bmj.e6409 (2012).

23. Ouyang, P. et al. Randomized trial of hormone therapy in women after coronary bypass surgery. Evidence of differential effect of hormone therapy on angiographic progression of disease in saphenous vein grafts and native coronary arteries. Atherosclerosis 189, 375-386. https://doi.org/10.1016/j.atherosclerosis.2005.12.015 (2006).

24. Hodis, H. N. et al. Estrogen in the prevention of atherosclerosis. A randomized, double-blind, placebo-controlled trial. Ann. Intern. Med. 135, 939-953. https://doi.org/10.7326/0003-4819-135-11-200112040-00005 (2001).

25. Veerus, P. et al. Results from the Estonian postmenopausal hormone therapy trial [ISRCTN35338757]. Maturitas 55, 162-173. https://doi.org/10.1016/j.maturitas.2006.01.012 (2006).

26. Herrington, D. M. et al. Effects of estrogen replacement on the progression of coronary-artery atherosclerosis. N. Engl. J. Med. 343, 522-529. https://doi.org/10.1056/NEJM200008243430801 (2000).

27. Hoibraaten, E. et al. Increased risk of recurrent venous thromboembolism during hormone replacement therapy-results of the randomized, double-blind, placebo-controlled estrogen in venous thromboembolism trial (EVTET). Thromb. Haemost. 84, 961-967 (2000).

28. Greenspan, S. L., Resnick, N. M. \& Parker, R. A. The effect of hormone replacement on physical performance in communitydwelling elderly women. Am. J. Med. 118, 1232-1239. https://doi.org/10.1016/j.amjmed.2005.03.004 (2005).

29. Gallagher, J. C., Fowler, S. E., Detter, J. R. \& Sherman, S. S. Combination treatment with estrogen and calcitriol in the prevention of age-related bone loss. J. Clin. Endocrinol. Metab. 86, 3618-3628. https://doi.org/10.1210/jcem.86.8.7703 (2001).

30. Waters, D. D. et al. Effects of hormone replacement therapy and antioxidant vitamin supplements on coronary atherosclerosis in postmenopausal women: a randomized controlled trial. JAMA 288, 2432-2440. https://doi.org/10.1001/jama.288.19.2432 (2002).

31. Hodis, H. N. et al. Hormone therapy and the progression of coronary-artery atherosclerosis in postmenopausal women. $\mathrm{N} \mathrm{Engl} \mathrm{J}$ Med 349, 535-545. https://doi.org/10.1056/NEJMoa030830 (2003).

32. Collins, P. et al. Randomized trial of effects of continuous combined HRT on markers of lipids and coagulation in women with acute coronary syndromes: WHISP pilot study. Eur. Heart J. 27, 2046-2053. https://doi.org/10.1093/eurheartj/ehl183 (2006).

33. Vickers, M. R. et al. Main morbidities recorded in the women's international study of long duration oestrogen after menopause (WISDOM): a randomised controlled trial of hormone replacement therapy in postmenopausal women. BMJ 335, 239. https:// doi.org/10.1136/bmj.39266.425069.AD (2007).

34. Grady, D. et al. Cardiovascular disease outcomes during 6.8 years of hormone therapy: Heart and Estrogen/progestin Replacement Study follow-up (HERS II). JAMA 288, 49-57. https://doi.org/10.1001/jama.288.1.49 (2002).

35. Hulley, S. et al. Noncardiovascular disease outcomes during 6.8 years of hormone therapy: heart and estrogen/progestin replacement study follow-up (HERS II). JAMA 288, 58-66. https://doi.org/10.1001/jama.288.1.58 (2002).

36. Manson, J. E. et al. Menopausal hormone therapy and health outcomes during the intervention and extended poststopping phases of the Women's Health Initiative randomized trials. JAMA 310, 1353-1368. https://doi.org/10.1001/jama.2013.278040 (2013).

37. Tierney, M. C. et al. A randomized double-blind trial of the effects of hormone therapy on delayed verbal recall in older women. Psychoneuroendocrinology 34, 1065-1074. https://doi.org/10.1016/j.psyneuen.2009.02.009 (2009).

38. Gleason, C. E. et al. Effects of hormone therapy on cognition and mood in recently postmenopausal women: findings from the randomized, controlled KEEPS-cognitive and affective study. PLoS Med. 12, e1001833. https://doi.org/10.1371/journal.pmed.10018 33 (2015) (discussion e1001833).

39. Clarke, S. C., Kelleher, J., Lloyd-Jones, H., Slack, M. \& Schofiel, P. M. A study of hormone replacement therapy in postmenopausal women with ischaemic heart disease: the Papworth HRT atherosclerosis study. BJOG 109, 1056-1062 (2002).

40. Nachtigall, L. E., Nachtigall, R. H., Nachtigall, R. D. \& Beckman, E. M. Estrogen replacement therapy II: a prospective study in the relationship to carcinoma and cardiovascular and metabolic problems. Obstet. Gynecol. 54, 74-79. https://doi.org/10.1097/00006 250-197907000-00017 (1979).

41. Hall, G., Pripp, U., Schenck-Gustafsson, K. \& Landgren, B. M. Long-term effects of hormone replacement therapy on symptoms of angina pectoris, quality of life and compliance in women with coronary artery disease. Maturitas 28, 235-242. https://doi. org/10.1016/s0378-5122(97)00080-7 (1998).

42. Constantine, G. D. et al. Endometrial safety of low-dose vaginal estrogens in menopausal women: a systematic evidence review. Menopause 26, 800-807. https://doi.org/10.1097/GME.0000000000001315 (2019).

43. Bhupathiraju, S. N. et al. Vaginal estrogen use and chronic disease risk in the Nurses' Health Study. Menopause 26, 603-610. https ://doi.org/10.1097/GME.0000000000001284 (2018).

44. Crandall, C. J. et al. Breast cancer, endometrial cancer, and cardiovascular events in participants who used vaginal estrogen in the Women's Health Initiative Observational Study. Menopause 25, 11-20. https://doi.org/10.1097/GME.0000000000000956 (2018).

45. Chaturvedi, N. Ethnic differences in cardiovascular disease. Heart 89, 681-686. https://doi.org/10.1136/heart.89.6.681 (2003).

46. Gold, E. B. et al. Factors related to age at natural menopause: longitudinal analyses from SWAN. Am. J. Epidemiol. 178, 70-83. https://doi.org/10.1093/aje/kws421 (2013).

47. Lutsey, P. L. et al. Atrial fibrillation and venous thromboembolism: evidence of bidirectionality in the Atherosclerosis Risk in Communities Study. J. Thromb. Haemost. 16, 670-679. https://doi.org/10.1111/jth.13974 (2018).

48. Moher, D., Liberati, A., Tetzlaff, J., Altman, D. G. \& The PRISMA Group. Preferred reporting items for systematic reviews and metaanalyses: the PRISMA statement. PLoS Med. 6(7), e1000097. https://doi.org/10.1371/journal.pmed1000097 (2009).

\section{Acknowledgements}

This research was supported by grant of the SNUH Research Fund (23-2017-0040), the Cancer Research Institute (0431-20190010), Seoul National University Hospital (2019-2020), the Ministry of Education of the Republic of Korea and the National Research Foundation of Korea (NRF-2018R1A2A3075397), and the Korea Health Technology R\&D Project through the Korea Health Industry Development Institute (KHIDI), funded by the Ministry of Health \& Welfare, Republic of Korea (HI19C1178). The author JEK, JC, and JYP received a scholarship from the BK21-plus education program.

\section{Author contributions}

J.E.K. and J.Y.C. contributed to the conception and design of the work, acquisition, analysis, and interpretation of data, and drafting of the article. J.H.C. and M.J.J. contributed to the acquisition, analysis, and interpretation of data. J.C., J.Y.P., A.S., S.M.P., and D.K. contributed to the interpretation of data, and critically revised the 
manuscript for important intellectual content. CB contributed to the drafting of the article. All the authors approved the final version.

\section{Competing interests}

The authors declare no competing interests.

\section{Additional information}

Supplementary information is available for this paper at https://doi.org/10.1038/s41598-020-77534-9.

Correspondence and requests for materials should be addressed to J.-Y.C.

Reprints and permissions information is available at www.nature.com/reprints.

Publisher's note Springer Nature remains neutral with regard to jurisdictional claims in published maps and institutional affiliations.

(c) (i) Open Access This article is licensed under a Creative Commons Attribution 4.0 International License, which permits use, sharing, adaptation, distribution and reproduction in any medium or format, as long as you give appropriate credit to the original author(s) and the source, provide a link to the Creative Commons licence, and indicate if changes were made. The images or other third party material in this article are included in the article's Creative Commons licence, unless indicated otherwise in a credit line to the material. If material is not included in the article's Creative Commons licence and your intended use is not permitted by statutory regulation or exceeds the permitted use, you will need to obtain permission directly from the copyright holder. To view a copy of this licence, visit http://creativecommons.org/licenses/by/4.0/.

(C) The Author(s) 2020 